\title{
Multiple pulmonary nodules mimicking metastasis in a case of systemic amyloidosis
}

Kaewkamol Srichai, M.D. ${ }^{(1)}$

Warath Chantaksinopas, M.D. ${ }^{(1)}$

Papon Prettiwitayakul, M.D. ${ }^{(1)}$

Kanet Kanjanapradit, M.D. ${ }^{(2)}$

From ${ }^{(1)}$ Department of Radiology, Faculty of Medicine, Prince of Songkla University, Songkhla, Thailand.

${ }^{(2)}$ Department of Pathology, Faculty of Medicine, Prince of Songkla University, Songkhla, Thailand.

Address correspondence to K.S. (e-mail: drkaew021@gmail.com)

\section{Abstract}

Amyloidosis is a disorder resulting from the abnormal accumulation of amyloid, a fibrillary protein, in various tissues and organs. Pulmonary amyloidosis can be a part of a systemic process and can mimic other lung diseases which present with multiple pulmonary masses or nodules, such as metastasis.

We reported a case of systemic amyloidosis, which is histopathologically confirmed from the nasal lesion, initially presented with multiple lung nodules mimicking pulmonary metastasis but had been stable for years. Pathological study of tissues obtained from three times of percutaneous transthoracic needle biopsy (PTNB) failed to show specific features. The findings on chest radiographs, contrast enhanced thoracic computed tomography (CT) and histology were reviewed and discussed.

Keywords: Amyloidosis, Pulmonary amyloidosis, Systemic amyloidosis, Chest CT, Pulmonary metastasis. 


\section{Introduction}

Amyloidosis is an uncommon systemic disorder that is characterized by extracellular accumulation and deposition of abnormal protein and protein derivatives $[1,2]$. No clear genetic or environmental factors in individual susceptibility to amyloid deposition have been made [3]. Amyloidosis can be systemic or localized. Systemic amyloidosis is subclassified into a primary (idiopathic) form and a secondary (reactive) form. Tuberculosis, familial Mediterranean fever, rheumatoid arthritis, or multiple myeloma are the causes of secondary amyloidosis [4]. The disease becomes clinically significant when its diffuse form affects organ function by replacing the normal cell structure [1]. The most commonly involved organs in patients with systemic amyloidosis are the heart and kidneys, followed by the nervous system, soft tissue, lungs, and liver. The involvement of the lungs is relatively common but rarely symptomatic. The most common symptoms of amyloid involvement with the respiratory system are coughs, wheezing, dyspnea on exertion, and hemoptysis $[4,5]$.

\section{Case summary}

A 61-year-old Thai female presented with abdominal discomfort for 2 months with mild pelvic pain, loss of appetite, constipation, and insignificant low-grade fever. No gynecologic symptoms were recorded. Her underlying disease included hypertension and GERD. She also underwent left salphingo-oophorectomy due to benign condition. Her physical examination revealed no fever and unremarkable vital signs. The abdominal and gynecologic examinations showed a low midline surgical scar without abnormal mass. Small-sized cervix, and normal uterus were noted. Her laboratory result revealed a low hemoglobin level $(10.7 \mathrm{~g} / \mathrm{dL})$ but a normal white cell count and platelets. The liver and renal functions as well as urinalysis were unremarkable. The routine chest radiograph showed multiple lung nodules which were varying in size (Figure 1). The contrast enhanced abdominal CT scan showed an ill-defined heterogeneous mass at the anterior aspect of the uterus (Figure 2). The contrast enhanced chest CT scan revealed multiple enhanced spiculated and well-defined nodules scattered in both lungs (Figure 3). Her diagnosis was the pelvic mass with lung metastasis, suspicious of uterine sarcoma. 


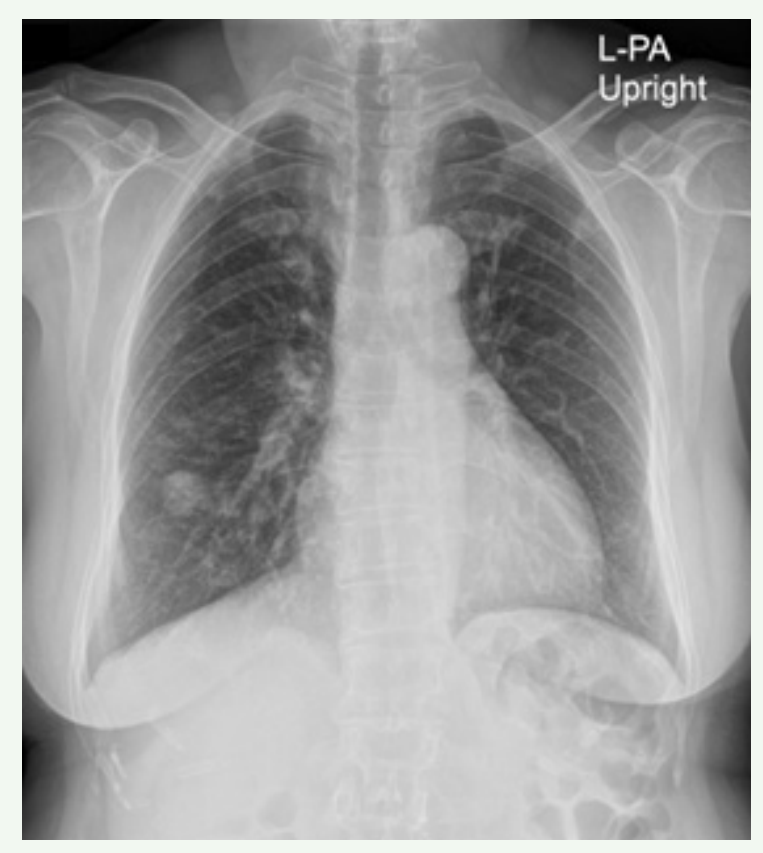

Figure 1: The frontal chest radiograph shows a well-defined nodule in the right lower lung field. A few smaller nodules in both upper lobes are also detected.
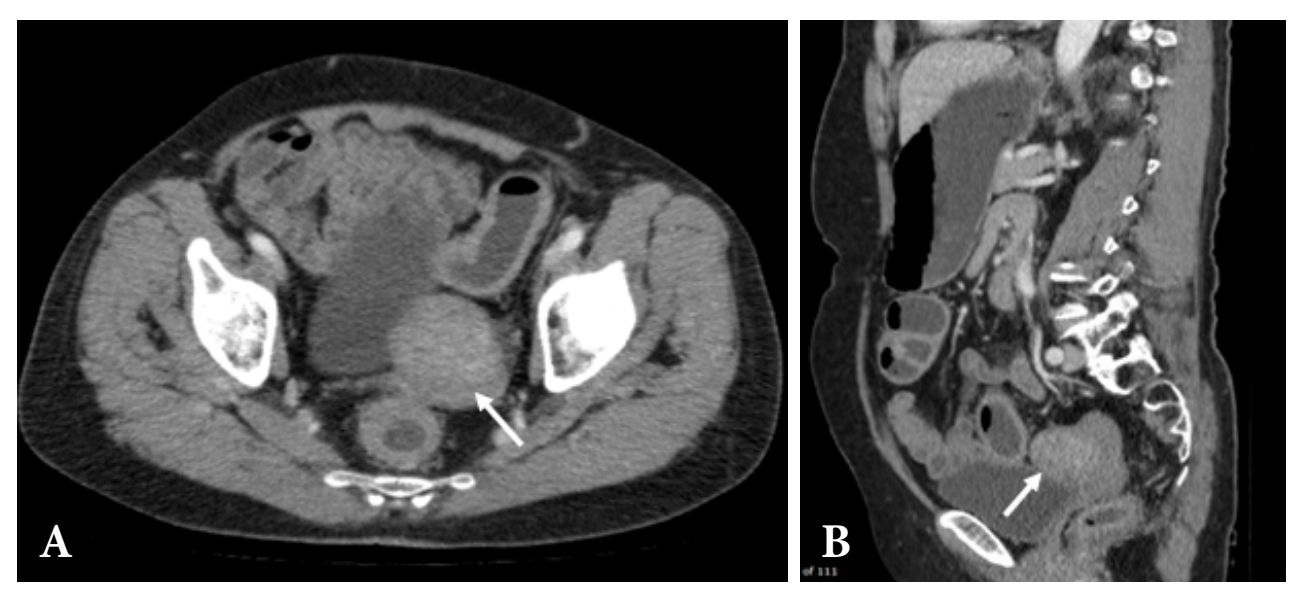

Figure 2: Contrast enhanced abdominal CT scan in axial (A) and reformatted sagittal (B) images show an ill-defined homogeneous mass at anterior aspect of the uterine fundus (arrow). 


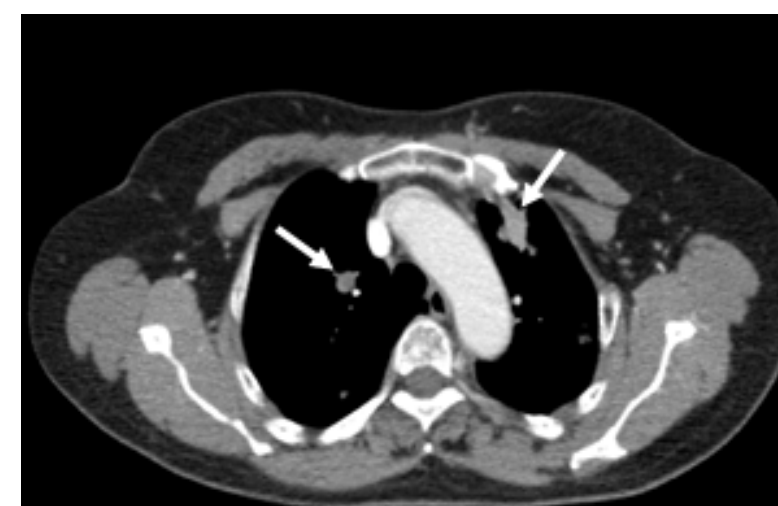

A
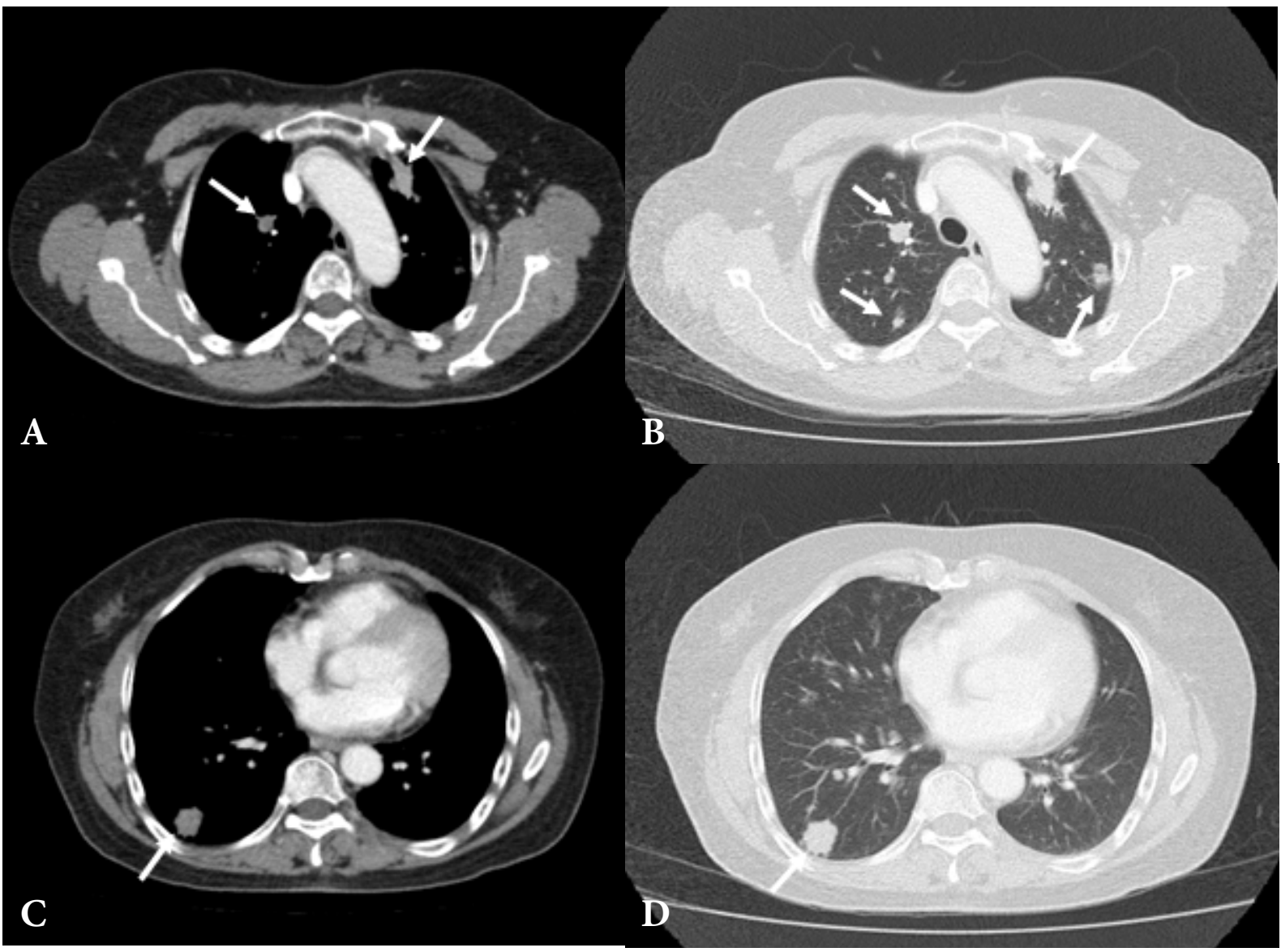

Figure 3: Contrast enhanced chest CT scan in mediastinal and lung windows at the level of aortic arch $(A, B)$ and at the level of inferior pulmonary veins $(C, D)$ show multiple heterogeneously enhanced spiculated and well-defined nodules scattered in both lungs which has no zonal predominance (arrow). These nodules are vary in size. No internal calcification or necrotic portion is observed.

The patient underwent transvaginal ultrasonography which revealed $6.6 \times 4.3 \mathrm{~cm}$ uterus with a small subserous myoma at the posterior wall and thin endometrium. Both ovaries were not visualized. Because of benign ultrasonographic appearance, the gynecologist suggested tissue diagnosis from the lung instead of the uterus. 
Percutaneous transthoracic needle biopsy (PTNB) was done at the largest lesion in the right lower lobe but the pathological result reported only small fragments of benign tissue; therefore, repeat biopsy was suggested. However, the patient lost follow-up.

Nearly two years later, the patient revisited our hospital, without any abnormal symptoms. She was transferred to the gynecologic department. Endometrial biopsy was done but the tissue was not sufficient for diagnosis while the Papanicolaou test showed a negative result for malignancy. The chest CT scans were done to re-evaluate disease progression, which showed rather stable nodules in both lungs. The second PTNB was done. The result was positive for glycoprotein material; periodic acid-Schiff (PAS)-positive, PAS with predigestion with diastase (PASD)-non-reactive, Congo red-negative; therefore, pathologist suspected pulmonary alveolar proteinosis (PAP). The patient was then referred to the chest unit.

After reviewed of all images, the PTNB was done for the third time to determine pulmonary metastasis, while the lesion at the uterus might be an asymptomatic myoma uteri due to its stable nature. The third PTNB showed a small fragment of benign lung tissue with chronic inflammation and tissue necrosis without a definite area of carcinoma. The pathological diagnosis was pulmonary alveolar proteinosis.

A year later, the patient developed left-sided nasal swelling. The physical examination revealed left lateral nasal ala swelling, hard consistency, and irregular skin tag. Because the symptom persisted after she received adequate topical medical treatment, a biopsy was performed. The specimen showed the deposition of eosinophilic amorphous material in the submucosal tissue with the displaced capillary lumen. The additional Congo red stain of the specimen showed apple-green birefringence under the polarized microscope (Figure 4). Therefore, the pathologic findings were compatible with amyloidosis. The chest unit was notified, and the possibility of systemic amyloidosis was discussed. 


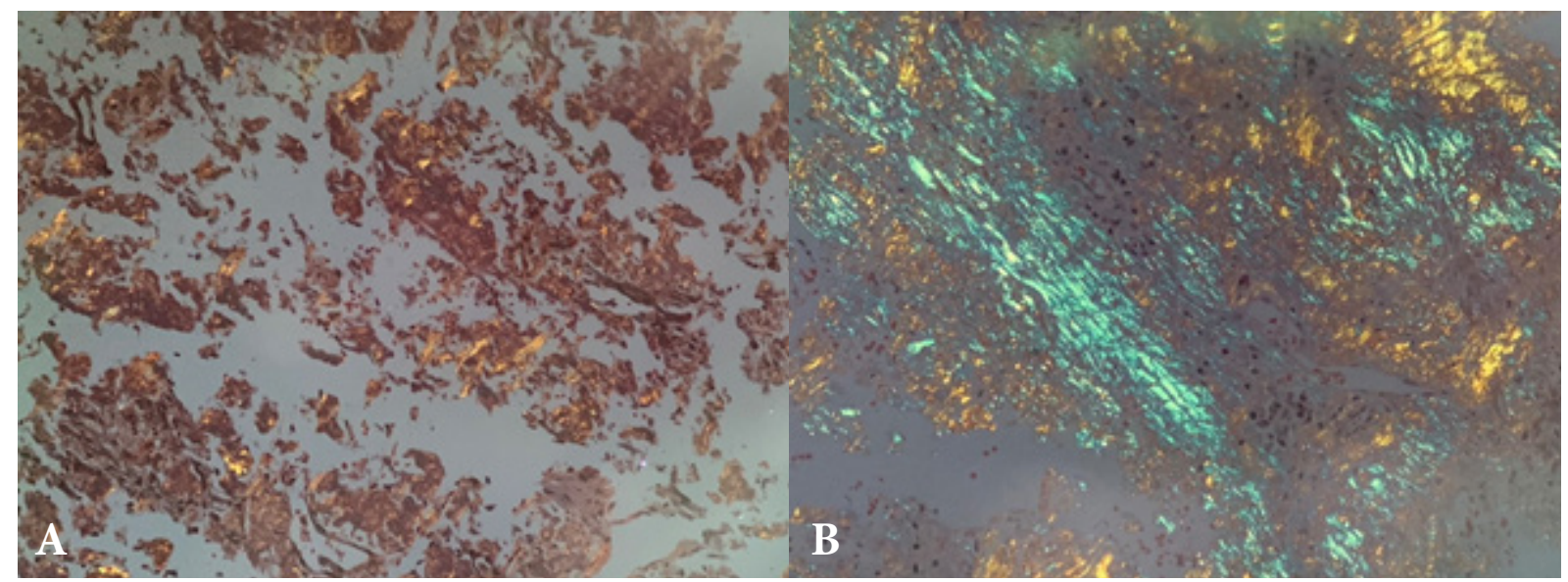

Figure 4: Specimen from the left nasal alar surgical biopsy with Congo red staining (A) shows the deposition of eosinophilic amorphous material in the submucosal tissue which also shows apple-green birefringence under the polarized microscope (B).

\section{Diagnosis :}

1. Systemic amyloidosis with nasal and pulmonary involvement.

2. Asymptomatic myoma uteri

\section{Discussion}

Three different forms of amyloidosis in the lungs from the pathologist's perspective include nodular pulmonary amyloidosis, diffuse alveolar-septal amyloidosis and tracheobronchial amyloidosis [5]. In Zhang et al[6] study, there is no specific clinical findings, examination, laboratory tests and radiological results for the diagnosis of nodular parenchymal pulmonary amyloidosis. The specimen with amyloid deposit shows apple-green birefringence when stained with Congo red and viewed under polarized light $[1,6]$.

In our study, the pulmonary findings revealed multiple nodules in both lungs which were stable after a follow-up on CT scan and CXR. During metastatic work up, the patient developed hard consistency irregular skin tag at the left lateral nasal alar. The final histopathological report was consistent with amyloidosis. Although 
the pathological result from the second PTNB was suspected of PAP but both PAP and amyloid disease may have common disturbances in immunoregulatory mechanisms as an important step in the pathogenesis [7]. In conclusion, pulmonary nodular amyloidosis was considered in this case, mimicking primary pulmonary or metastatic neoplasms.

In the previous studies, multiple deposits are commonly found in nodular parenchymal amyloid rather than focal deposits. Calcification is seen in about $50 \%$ of the cases, often centrally or in an irregular pattern within the nodule. The nodules may be of multiple shapes, sizes varying from 0.5 to $15 \mathrm{~cm}$ and with slow growth, often over years, and no regression [4]. CT can suggest the diagnosis because it is a good tool to demonstrate subtle calcification, which is often found in amyloidosis. The typical locations are peripherally or subpleural. Cavitation is very rare [4].

The hyperdensity of the multiple pulmonary nodules compared to muscle in our case, showed no clear explanation. They could be parts of calcification which is an important characteristic that can be found in almost half of the cases or increased enhancement which is suspected from morphologic alterations from amyloid deposition $[4,11]$. However, the histopathological report revealed no calcification or structural alteration, such as neovascularization orgranulation tissue, that can elucidate increased contrast enhancement. Eventually, the lesions could be hyperattenuated on unenhanced $\mathrm{CT}$, which are observed in the brain [12]. However, no plain CT in our study was performed.

The cases reported by Zhang et al [6] and Seo et al [8], exhibited multiple lung nodules of pulmonary amyloidosis with moderate 18F-FDG uptake. Positive results of 18F-FDG PET/CT on pulmonary nodules should be interpreted with caution in differentiating pulmonary nodular amyloidosis from malignant lesions. Histological confirmation is required for the final diagnosis. CTguided PTNB should be considered due to a less invasive nature than open lung biopsy. Although the diagnosis of amyloidosis should be based on tissue biopsy, in our study, three times of PTNB showed negative results. Searching amyloid in 
more than one tissue and using different methods can exclude the possibility for false positive and false negative results and gives an idea for the heaviness and spread of the process [9].

Nodular parenchymal amyloidosis does not require a specific treatment. The surgical resection may be required if a large nodule causes a mass or spaceoccupying effect $[6,10]$.

In conclusion, pulmonary nodular amyloidosis is a rare and usually localized disease that can mimic other pulmonary disorders, such as neoplastic and granulomatous processes. As such, this condition should be considered in the differential diagnosis of pulmonary nodules or masses. 


\section{References}

1. Wechalekar AD, Gillmore JD, Hawkins PN. Systemic amyloidosis. Lancet 2016;387:2641-54.

2. Gillmore JD, Hawkins PN. Amyloidosis and the respiratory tract. Thorax 1999;54:444-51.

3. Thompson PJ, Citron KM. Amyloid and the lower respiratory tract. Thorax 1983;38:84-7.

4. Urban BA, Fishman EK, Goldman SM, Scott WW, Jones B, Humphrey RL, et al. CT evaluation of amyloidosis: spectrum of disease. Radiographics 1993;13:1295-308.

5. Milani P, Basset M, Russo F, Foli A, Palladini G, Merlini G. The lung in amyloidosis. Eur Respir Rev 2017;26.pii: 170046. doi: 10.1183/16000617.

6. Zhang LN, Xue XY, Wang N, Wang JX. Mimicking pulmonary multiple metastatic tumors: A case of primary nodular parenchymal pulmonary amyloidosis with review of the literature. Oncol Lett 2012;4:1366-70.

7. Merino-Angulo A, Pérez-Martí M, Díaz de Otazu R. Pulmonary alveolar phospholipoproteinosis associated with amyloidosis. Chest 1990;98:1048.

8. Seo JH, Lee SW, Ahn BC, Lee J. Pulmonary amyloidosis mimicking multiple metastatic lesions on F-18 FDG PET/CT. Lung Cancer 2010;67:376-9.

9. Bogov B, Lubomirova M, Kiperova B. Biopsy of subcutaneus fatty tissue for diagnosis of systemic amyloidosis. Hippokratia 2008;12:236-9. 
10. Lee SC, Johnson H. Multiple nodular pulmonary amyloidosis. A case report and comparison with diffuse alveolar-septal pulmonary amyloidosis. Thorax 1975;30:178-85.

11. Czeyda-Pommersheim F, Hwang M, Chen SS, Strollo D, Fuhrman C, Bhalla S. Amyloidosis: Modern cross-sectional Imaging. RadioGraphics 2015;35: 1381-92.

12. Parmar H, Rath T, Castillo M, Gandhi D. Imaging of focal amyloid depositions in the head, neck, and spine: amyloidoma. AJNR Am J Neuroradiol 2010;31:1165-70. 